\title{
Distance Between Bipolar Fuzzy Sets
}

\author{
M. Rajeshwari ${ }^{1 *}$ \\ ${ }^{1}$ Department of Mathematics, \\ School of Engineering, Presidency University, \\ Bangalore, India.
}

\author{
R. Murugesan ${ }^{2}$ \\ ${ }^{2}$ Department of Mathematics, \\ Reva University, \\ Bangalore.
}

\author{
K. A. Venkatesh ${ }^{3}$ \\ ${ }^{3}$ Department of Mathematics and Comp. Science, \\ Myanmar Institute of Information Technology, \\ Myanmar.
}

\begin{abstract}
An geometrical representational of bipolar fuzzy set is a point of going of our proposition of distance between bipolar fuzzy sets. Acquainted another definition furthermore compared those methodology utilized on fuzzy set also intuitionistic fuzzy set.
\end{abstract}

Keywords: Fuzzy set, Intuitionistic fuzzy set, bipolar fuzzy set.

\section{INTRODUCTION}

Crisp data cannot be used for most of the real time problems. The various type of uncertainties problems cannot be solved by traditional method. Fuzzy arrangement of presented by Zadeh in 1965, many methodologies and hypotheses treating imprecision and uncertainty has been proposed. Bipolar fuzzy set hypothesis is one of the way to deal with handle imprecision and uncertainty in various ways. Bipolar fuzzy set hypothesis is an expansion of fuzzy set hypothesis.

In this paper we presented the new distance measure of bipolar fuzzy set and looked at the approach utilized on fuzzy set and intuitionistic fuzzy set. The four fundamental distance in the bipolar fuzzy set is Hamming distance, normalized Hamming distance, Euclidean distance and normalized Euclidean distance.

\section{PRELIMINARIES}

Definition 2.1:[13] A fuzzy set $\mathrm{A}$ in $\mathrm{X}=\{\mathrm{x}\}$ is given by $A=\left\{\left(x, \mu_{A}(x)\right) / x \in X\right\}$ where $\mu_{A}: X \rightarrow[0,1]$ is the membership of the fuzzy set A, $\mu_{A}(x) \in[0,1]$ the membership of $x \in X$ in A.

Definition 2.2:[11] An intuitionistic fuzzy set $\mathrm{A}$ in $\mathrm{X}$ is given by $A=\left\{\left(x, \mu_{A}(x), v_{A}(x)\right) / x \in X\right\}$ where $\mu_{A}: X \rightarrow[0,1]$, $v_{A}: X \rightarrow[0,1]$ with the condition $0 \leq \mu_{A}(x)+v_{A}(x) \leq 1, \forall x \in X$. The numbers $\mu_{A}(x), v_{A}(x) \in[0,1]$ denote the degree of membership and non-membership of $\mathrm{x}$ to A.

The amount $\pi_{A}=1-\mu_{A}(x)-v_{A}(x)$ is called the hesitation part or intuitionistic index of $\mathrm{x}$ in $\mathrm{A}$.

Definition 2.3:[10] An Bipolar fuzzy set $\mathrm{A}$ in $\mathrm{X}$ is given by $A=\left\{\left(x, \mu_{A}^{P}(x), \mu_{A}^{N}(x)\right) / x \in X\right\}$ where $\mu_{A}^{P}: X \rightarrow[0,1]$, $\mu_{A}^{N}: X \rightarrow[-1,0]$.The positive membership degree $\mu_{A}^{P}$ and the negative membership degree $\mu_{A}^{N}$ denotes the satisfaction degree of an element $X$ to the property corresponding to bipolar valued fuzzy set.

Geometrical insertion about bipolar fuzzy set indicated on fig. 1.In the form $\left(\mu_{A}^{P}(x), \mu_{A}^{N}(x)\right)$ is the representation of positive values and the representation of negative values. 


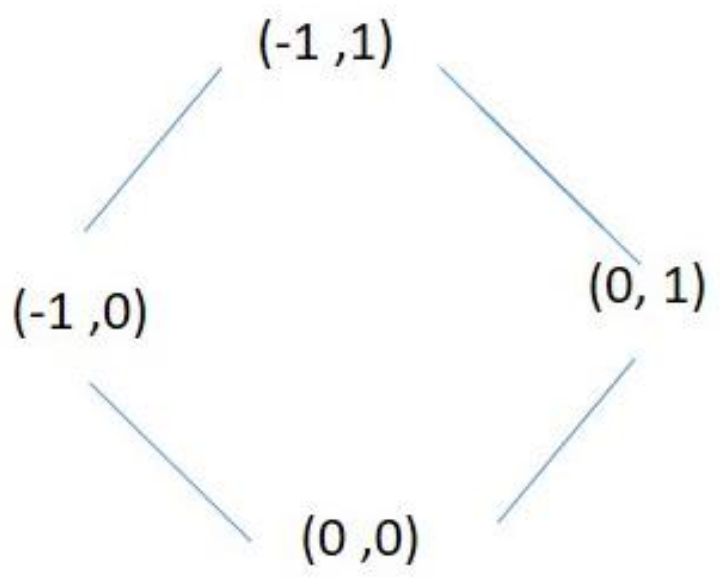

\section{Positive value}

\section{Negative value}

Fig. 1. Geometrical interpolation of bipolar fuzzy set

\section{DISTANCE IN FUZZY SET}

The distance of Hamming, normalized Hamming, Euclidean and normalized Euclidean form are used to measures the distance between the set in the same universe.

- The distance of Hamming is $\lambda(E, F)$

$$
\lambda(E, F)=\sum_{k=1}^{n}\left|\mu_{E}\left(q_{k}\right)-\mu_{F}\left(q_{k}\right)\right|
$$

- The distance of normalized Hamming is $\delta(E, F)$

$$
\delta(E, F)=\frac{1}{n} \sum_{k=1}^{n}\left|\mu_{E}\left(q_{k}\right)-\mu_{F}\left(q_{k}\right)\right|
$$

- The distance of Euclidean is $\psi(E, F)$

$\psi(E, F)=\sqrt{\sum_{k=1}^{n}\left(\mu_{E}\left(q_{k}\right)-\mu_{F}\left(q_{k}\right)\right)^{2}}$

- The distance of normalized Euclidean is $\sigma(E, F)$

$\sigma(E, F)=\sqrt{\frac{1}{n} \sum_{k=1}^{n}\left(\mu_{E}\left(q_{k}\right)-\mu_{F}\left(q_{k}\right)\right)^{2}}$

Only the positive membership value are present in the above formula.

\section{COMPARISON OF INTUITIONISTIC FUZZY SET AND BIPOLAR FUZZY SET AND THEIR DISTANCE}

We compare bipolar fuzzy set with an intuitionistic fuzzy set under the conditions $\mu_{E}^{P}(q)=\mu_{E}(q), \mu_{E}^{N}(q)=-v_{E}(q)$. In bipolar fuzzy set the hamming distance $\lambda^{\prime}(E, F)$ is a representation of intuitionistic fuzzy set and fuzzy set. 


$$
\begin{aligned}
\lambda^{\prime}(E, F) & =\sum_{k=1}^{n}\left(\left|\mu_{E}^{P}\left(q_{k}\right)-\mu_{F}^{P}\left(q_{k}\right)\right|+\left|\mu_{E}^{N}\left(q_{k}\right)-\mu_{F}^{N}\left(q_{k}\right)\right|\right) \\
& =\sum_{k=1}^{n}\left(\left|\mu_{E}^{P}\left(q_{k}\right)-\mu_{F}^{P}\left(q_{k}\right)\right|+\left|-v_{E}\left(q_{k}\right)+v_{F}\left(q_{k}\right)\right|\right) \\
& =\sum_{k=1}^{n}\left(\left|\mu_{E}^{P}\left(q_{k}\right)-\mu_{F}^{P}\left(q_{k}\right)\right|+\left|-1+\mu_{E}\left(q_{k}\right)+1-\mu_{F}\left(q_{k}\right)\right|\right) \\
& =\sum_{k=1}^{n}\left(\left|\mu_{E}^{P}\left(q_{k}\right)-\mu_{F}^{P}\left(q_{k}\right)\right|+\left|\mu_{E}^{P}\left(q_{k}\right)-\mu_{F}^{P}\left(q_{k}\right)\right|\right) \\
& =2 \sum_{k=1}^{n}\left(\left|\mu_{E}\left(q_{k}\right)-\mu_{F}\left(q_{k}\right)\right|\right) \\
& =2 \lambda(E, F)
\end{aligned}
$$

It is twice vast as hamming distance of fuzzy set.

In bipolar fuzzy set the normalized Hamming distance $\delta^{\prime}(E, F)$ is a representation of intuitionistic fuzzy set and fuzzy set.

$$
\begin{aligned}
\delta^{\prime}(E, F) & =\frac{1}{n} \sum_{k=1}^{n}\left(\left|\mu_{E}^{P}\left(q_{k}\right)-\mu_{F}^{P}\left(q_{k}\right)\right|+\left|\mu_{E}^{N}\left(q_{k}\right)-\mu_{F}^{N}\left(q_{k}\right)\right|\right) \\
& =\frac{1}{n} \sum_{k=1}^{n}\left(\left|\mu_{E}^{P}\left(q_{k}\right)-\mu_{F}^{P}\left(q_{k}\right)\right|+\left|-v_{E}\left(q_{k}\right)+v_{F}\left(q_{k}\right)\right|\right) \\
& =\frac{1}{n} \sum_{k=1}^{n}\left(\left|\mu_{E}^{P}\left(q_{k}\right)-\mu_{F}^{P}\left(q_{k}\right)\right|+\left|-1+\mu_{E}\left(q_{k}\right)+1-\mu_{F}\left(q_{k}\right)\right|\right) \\
& =\frac{1}{n} \sum_{k=1}^{n}\left(\left|\mu_{E}^{P}\left(q_{k}\right)-\mu_{F}^{P}\left(q_{k}\right)\right|+\left|\mu_{E}^{P}\left(q_{k}\right)-\mu_{F}^{P}\left(q_{k}\right)\right|\right) \\
& =\frac{2}{n} \sum_{k=1}^{n}\left(\left|\mu_{E}\left(q_{k}\right)-\mu_{F}\left(q_{k}\right)\right|\right) \\
& =\frac{2}{n} \delta(E, F)
\end{aligned}
$$

It is two times multiplied as normalized hamming distance of a fuzzy set.

In bipolar fuzzy set the Euclidean distance $\psi^{\prime}(E, F)$ is a representation of intuitionistic fuzzy set and fuzzy set, is equal to

$$
\begin{aligned}
\psi^{\prime}(E, F) & =\sqrt{\sum_{k=1}^{n}\left(\left(\mu_{E}^{P}\left(q_{k}\right)-\mu_{F}^{P}\left(q_{k}\right)\right)^{2}+\left(\mu_{E}^{N}\left(q_{k}\right)-\mu_{F}^{N}\left(q_{k}\right)\right)^{2}\right)} \\
& =\sqrt{\sum_{k=1}^{n}\left(\left(\mu_{E}^{P}\left(q_{k}\right)-\mu_{F}^{P}\left(q_{k}\right)\right)^{2}+\left(-v_{E}\left(q_{k}\right)+v_{F}\left(q_{k}\right)\right)^{2}\right)} \\
& =\sqrt{\sum_{k=1}^{n}\left(\left(\mu_{E}^{P}\left(q_{k}\right)-\mu_{F}^{P}\left(q_{k}\right)\right)^{2}+\left(-1+\mu_{E}\left(q_{k}\right)+1-\mu_{F}\left(q_{k}\right)\right)^{2}\right)} \\
& =\sqrt{\sum_{k=1}^{n}\left(\left(\mu_{E}^{P}\left(q_{k}\right)-\mu_{F}^{P}\left(q_{k}\right)\right)^{2}+\left(\mu_{E}^{P}\left(q_{k}\right)-\mu_{F}^{P}\left(q_{k}\right)\right)^{2}\right)} \\
& =\sqrt{2 \sum_{k=1}^{n}\left(\mu_{E}\left(q_{k}\right)-\mu_{F}\left(q_{k}\right)\right)^{2}} \\
& =\sqrt{2} \psi(E, F)
\end{aligned}
$$

It is increased by $\sqrt{2}$ Euclidean distance of a fuzzy set. 
In bipolar fuzzy set the normalized Euclidean distance $\sigma^{\prime}(E, F)$ is a representation of intuitionistic fuzzy set and fuzzy set, is equal to

$$
\begin{aligned}
\sigma^{\prime}(E, F) & =\sqrt{\frac{1}{n} \sum_{k=1}^{n}\left(\left(\mu_{E}^{P}\left(q_{k}\right)-\mu_{F}^{P}\left(q_{k}\right)\right)^{2}+\left(\mu_{E}^{N}\left(q_{k}\right)-\mu_{F}^{N}\left(q_{k}\right)\right)^{2}\right)} \\
& =\sqrt{\frac{1}{n} \sum_{k=1}^{n}\left(\left(\mu_{E}^{P}\left(q_{k}\right)-\mu_{F}^{P}\left(q_{k}\right)\right)^{2}+\left(-v_{E}\left(q_{k}\right)+v_{F}\left(q_{k}\right)\right)^{2}\right)} \\
& =\sqrt{\frac{1}{n} \sum_{k=1}^{n}\left(\left(\mu_{E}^{P}\left(q_{k}\right)-\mu_{F}^{P}\left(q_{k}\right)\right)^{2}+\left(-1+\mu_{E}\left(q_{k}\right)+1-\mu_{F}\left(q_{k}\right)\right)^{2}\right)} \\
& =\sqrt{\frac{1}{n} \sum_{k=1}^{n}\left(\left(\mu_{E}^{P}\left(q_{k}\right)-\mu_{F}^{P}\left(q_{k}\right)\right)^{2}+\left(\mu_{E}^{P}\left(q_{k}\right)-\mu_{F}^{P}\left(q_{k}\right)\right)^{2}\right)} \\
& =\sqrt{\frac{2}{n} \sum_{k=1}^{n}\left(\mu_{E}\left(q_{k}\right)-\mu_{F}\left(q_{k}\right)\right)^{2}} \\
& =\sqrt{\frac{2}{n}} \sigma(E, F)
\end{aligned}
$$

The normalized Euclidean distance of a fuzzy set is multiplied by $\sqrt{2}$ in Bipolar fuzzy set.

Example 1: Consider five fuzzy sets A, B, C, D and E in $\mathrm{X}=\{1\}$. The bipolar representation of them is $A=\left(\mu_{A}^{P}, \mu_{A}^{N}\right) / 1$, explified by $A=(1,0) / 1, B=(0,-1) / 1, C=\left(\frac{1}{4}, \frac{-3}{4}\right) / 1, D=\left(\frac{1}{2}, \frac{-1}{2}\right) / 1, E=\left(\frac{3}{4}, \frac{-1}{4}\right) / 1$.

The normal representation of fuzzy set for Euclidean distance is calculated as,

$$
\begin{aligned}
& \psi(C, E)=\sqrt{\left(\frac{1}{4}-\frac{3}{4}\right)^{2}}=\frac{1}{2} \\
& \psi(C, D)=\sqrt{\left(\frac{1}{4}-\frac{1}{2}\right)^{2}}=\frac{1}{4} \\
& \psi(C, A)=\sqrt{\left(\frac{1}{4}-1\right)^{2}}=\frac{3}{4} \\
& \psi(E, D)=\sqrt{\left(\frac{3}{4}-\frac{1}{2}\right)^{2}}=\frac{1}{4} \\
& \psi(D, A)=\sqrt{\left(\frac{1}{2}-1\right)^{2}}=\frac{1}{2} \\
& \psi(B, D)=\sqrt{\left(0-\frac{1}{2}\right)^{2}}=\frac{1}{2} \\
& \psi(B, A)=\sqrt{(0-1)^{2}}=1
\end{aligned}
$$

Now we calculate the Euclidean distance of bipolar fuzzy set, 


$$
\begin{aligned}
& \psi^{\prime}(C, E)=\sqrt{\left(\frac{1}{4}-\frac{3}{4}\right)^{2}+\left(-\frac{3}{4}+\frac{1}{4}\right)^{2}}=\frac{\sqrt{2}}{2} \\
& \psi^{\prime}(C, D)=\sqrt{\left(\frac{1}{4}-\frac{1}{2}\right)^{2}+\left(-\frac{3}{4}+\frac{1}{2}\right)^{2}}=\frac{\sqrt{2}}{4} \\
& \psi^{\prime}(C, A)=\sqrt{\left(\frac{1}{4}-1\right)^{2}+\left(-\frac{3}{4}+0\right)^{2}}=\frac{3 \sqrt{2}}{4} \\
& \psi^{\prime}(E, D)=\sqrt{\left(\frac{3}{4}-\frac{1}{2}\right)^{2}+\left(-\frac{1}{4}+\frac{1}{2}\right)^{2}}=\frac{\sqrt{2}}{4} \\
& \psi^{\prime}(D, A)=\sqrt{\left(\frac{1}{2}-1\right)^{2}+\left(-\frac{1}{2}+0\right)^{2}}=\frac{\sqrt{2}}{2} \\
& \psi^{\prime}(B, D)=\sqrt{\left(0-\frac{1}{2}\right)^{2}+\left(-1+\frac{1}{2}\right)^{2}}=\frac{\sqrt{2}}{2} \\
& \psi^{\prime}(B, A)=\sqrt{(0-1)^{2}+(-1+0)^{2}}=\sqrt{2}
\end{aligned}
$$

Therefore the distance of fuzzy set and bipolar fuzzy set are different and their essence is the same.

Example 2: Consider the two fuzzy set $\varsigma, \tau$ in $Z=\{1,2,3,4\}$. The bipolar representation of them is $\varsigma=\left(\mu_{\varsigma}^{P}, \mu_{\varsigma}^{N}\right) / 1$, explified by

$$
B=(0.1,-0.9) / 1+(0.2,-0.6) / 2+(0.7,-0.3) / 3+(0,-0.3) / 4 \text {. }
$$

$$
\varsigma=(0.2,-0.4) / 1+(0.6,-0.4) / 2+(0.8,-0.2) / 3+(0.4,-0.1) / 4
$$

The Hamming distance of fuzzy set is calculated as,

$$
\lambda(\varsigma, \tau)=|0.2-0.1|+|0.6-0.2|+|0.8-0.7|+|0.4-0|=1
$$

The Hamming distance $d^{\prime}(A, B)$ for bipolar fuzzy set is,

$$
\begin{aligned}
\lambda^{\prime}(\varsigma, \tau) & =|0.2-0.1|+|-0.4+0.9|+|0.6-0.2|+|-0.4+0.6|+|0.8-0.7|+|-0.2+0.3|+|0.4-0|+|-0.1+0.3| \\
& =2
\end{aligned}
$$

The normalized Hamming distance $\delta(\varsigma, \tau)$ for fuzzy set is,

$$
\delta(\varsigma, \tau)=\frac{1}{n} \lambda(\varsigma, \tau)=\frac{1}{4}
$$

The normalized Hamming distance $\delta^{\prime}(\varsigma, \tau)(\mathrm{A}, \mathrm{B})$ for Bipolar fuzzy set is,

$$
\delta^{\prime}(\varsigma, \tau)=\frac{1}{n} \lambda^{\prime}(\varsigma, \tau)=\frac{1}{2}
$$

The Euclidean distance of fuzzy set is,

$$
\psi(\varsigma, \tau)=\sqrt{(0.2-0.1)^{2}+(0.6-0.2)^{2}+(0.8-0.7)^{2}+(0.4-0)^{2}}=0.58309
$$

The Euclidean distance $e^{\prime}(\mathrm{A}, \mathrm{B})$ for Bipolar fuzzy set is

$$
\begin{aligned}
\psi^{\prime}(\varsigma, \tau) & =\sqrt{\begin{array}{l}
(0.2-0.1)^{2}+(-0.4+0.9)^{2}+(0.6-0.2)^{2}+(-0.4+0.6)^{2}+(0.8-0.7)^{2}+(-0.2+0.3)^{2}+(0.4-0)^{2} \\
+(-0.1+0.3)^{2}
\end{array}} \\
& =0.8246
\end{aligned}
$$

The normalized Euclidean distance of fuzzy set is,

$\sigma(\varsigma, \tau)=\sqrt{\frac{1}{4}} \psi(\varsigma, \tau)=0.291545$

The Euclidean distance $\sigma^{\prime}(\varsigma, \tau)$ for Bipolar fuzzy set is 
$\sigma^{\prime}(\varsigma, \tau)=\sqrt{\frac{1}{4}} \psi^{\prime}(\varsigma, \tau)=0.4123$

\section{RESULTS}

Taking to the account that the membership function for the distance calculated between two fuzzy set is

$\lambda(E, F) \leq n$,

$\delta(E, F) \leq 1$,

$\psi(E, F) \leq \sqrt{n}$,

$\sigma(E, F) \leq 1$,

Taking to the account that the membership function for the distance calculated between two bipolar fuzzy set is

$\lambda^{\prime}(E, F) \leq 2 n$,

$\delta^{\prime}(E, F) \leq 2$,

$\psi^{\prime}(E, F) \leq \sqrt{2 n}$,

$\sigma^{\prime}(E, F) \leq \sqrt{2}$.

\section{CONCLUSION}

In this paper we calculated the distance of bipolar fuzzy set by the representation of intuitionistic and fuzzy set and also we compared the distance of fuzzy set and bipolar fuzzy set.

\section{REFERENCES}

[1] K. Atanassov, Intuitionistic fuzzy sets, Fuzzy Sets and Systems 20 (1986) 87-96.

[2] Jin Han Park,Ki Moon Lim,jong seo Park and Young chel Kwun,Distance between interval-valued intuitionistic fuzzy set, Journal of physics: Conference series 96 (2008)

[3] K. Atanassov, New operations dened over the intuitionistic fuzzy sets, Fuzzy Sets and Systems 61 (1994) $137-142$.

[4] K. Atanassov, Operators over interval valued intuitionistic fuzzy sets, Fuzzy Sets and Systems 64 (1994) $159-174$.

[5] K. Atanassov, Intuitionistic Fuzzy Sets: Theory and Applications, Physica-Verlag, Heidelberg, 1999.

[6] J. Kacprzyk, Multistage Fuzzy Control, Wiley, Chichester, 1997.

[7] E. Szmidt, J. Kacprzyk, Distance between intuitionistic fuzzy sets, Fuzzy Sets and Systems 114 (2000) $505-518$.

[8] E. Szmidt, J. Kacprzyk, Intuitionistic fuzzy sets in group decision making, Notes on IFS 2 (1996) 15 - 32.

[9] E. Szmidt, J. Kacprzyk, Group decision making via intuitionistic fuzzy sets, Proc. FUBEST’96, Soa, Bulgaria, 1996 , pp. 107-112.

[10] K.M.Lee, Comparison of interval-valued fuzzy sets, intuitionistic fuzzy sets, and bipolar-valued fuzzy sets.

[11] E. Szmidt, J. Kacprzyk, Intuitionistic fuzzy sets for more realistic group decision making, Proc. TRANSITIONS'97, Warsaw, Poland, 1997, pp. 430 -433 .

[12] E. Szmidt, J. Kacprzyk, On measuring distances between intuitionistic fuzzy sets, Notes on IFS 3 (1997) 1-13.

[13] L.A. Zadeh, Fuzzy sets, Inform. and Control 8 (1965) 338 - 353. 